\title{
Design Developing an Early Model of Cat Robot for the Use of Early Treatment of Children with Autism Spectrum Disorder (ASD)
}

\author{
Keum Hi Mun ${ }^{1}$, Ja Young Kwon ${ }^{2}$, Bo Hee Lee ${ }^{3}$ and Jin Soon Jung ${ }^{4}$ \\ ${ }^{1}$ Department of Industrial Design, Semyung University, Jecheon-si, R. O. Korea, \\ ${ }^{2}$ Department of Social Welfare, Semyung University, Jecheon-si, R. O. Korea, \\ ${ }^{3}$ Department of Electronical Engineering, Semyung University, Jecheon-si, $R . O$. \\ Korea \\ ${ }^{4}$ Department of Fashion Design, Semyung University, Jecheon-si, R. O. Korea, \\ \{khmun, jykwon66, bhlee, bobejin\}@semyung.ac.kr
}

\begin{abstract}
The purpose of this research is to design develop an early model for the use of early treatment that will improve the social interactions of children with ASD. Researchers from the department of social welfare, electrical engineering, industrial design, and fashion design carried out a fusion research for this purpose. The department of social welfare conducted a survey with professional therapists in order to first find out the necessary treatment components of the cat robot. The department of electrical engineering gave technical suggestions on the practical functions of the robot such as movements and emotional exchanges with humans. In addition, the department of industrial design proposed the robot's exterior, movement, and character designs. Considering that the robot will be used as a therapeutic medium for children, they also developed an eco-friendly material in order to prevent infection and other hygienic problems and also improve the therapeutic effects of the robot. According to the result of FGI's analysis, elements of function and motion have to be prepared for a cat robot was grasped. In consideration of these elements, guidelines to the construction of shape and motions for the design of the cat robot were established. By applying these guidelines, the design of outer shape of the cat robot was developed. And a cat robot's structures, electrical circuits, and driving program for the treatment of children with ASD were suggested. Design of the cat robot's Outer shape has to be developed to match inside structure of the cat robot, and the early model of the cat robot has to be completed to match these criteria.
\end{abstract}

Keywords: Cat Robot, Treatment of ASD, Design Development, Early Model

\section{Introduction}

ASD is reportedly the fastest spreading childhood disorder (Autism Society of America, 2008). It is known that one in 150 children is diagnosed with ASD (Center for Disease Control, 2008). The main characteristics of children with ASD are that they have social interaction disabilities, communication disabilities, repetitive stereotypy behaviors, and limited attention spans. Existing methods of treatment and education are greatly limited because these children cannot properly express their own emotional states and consequently a new treatment system needs to be developed. A study on an animal shaped robot that has an animal-assisted therapy effect on early treatment for children with ASD, and has no dangerous elements of infection or allergy such as like a real animal, has been started in the late 90's. Therapeutic approaches of ASD that use robots commonly show that robots were 
able to draw out high levels of social interactions from the children with ASD (Cho, Kwon and Shin, 2009). [1] Robin et al., (2005) claimed that the children's social interactive time span increased as a result of a study about social interaction that was conducted with four children and humanoid robots. [2] According to another study conducted by Feil-Seifer and Matric (2008), children with children with ASD increased the level of social interactions with the robot as well as with their parents after being treated with robotic therapy. [3] There were several researches in Korea as well. The first one to apply robots in the treatment of developmental disorders was Cho, kwon, and Shin (2009). This research conducted one ten-minute session per week for eight weeks where adolescents with ASD interacted with humanoid robots. It was discovered that these adolescents interacted actively with the robots. Kim, et al., (2010) study on the response of autistic children through interactions with robots showed that robots have a positive effect on children such as increased eye contact, attention, and concentration with teachers. [4] It is established that an animal shaped robot is effective in the treatment for children with developmental disorders and has treatment efficiency. This study conducted FGIs with professional therapists and parents of children with ASD in order to learn the necessary or desirable motions and functionalities robots should have to be used for treating children with ASD. A cat is selected as an object animal of robot for an early treatment of children with ASD according to the result of their preference research. According to the results of FGI's analysis, elements of functions and motions should have to be prepared for the cat robot were grasped with elements of emotional expression, emotional stimulation, motional function, robot management, and in the material aspect.

Design for appearance, behaviour and character of a cat robot must be studied to design the cat robot for early treatment of children with ASD. The face, body, arms, legs and tail are belonged to the category of cat robot's appearance design. For a design of behaviour of cat robot, the cat's behaviour must be considered, but, 'hugging', 'eye contact', 'parrotry' and 'reactions to an inappropriate act' should be studied as well. For the use of early treatment of children with ASD, the character of cat robot needs to be personified for looking after rather a human's character than a cat's character. In order to design of the robot for the treatment of children with ASD, guidelines of design interaction for structure of shape and motions should be constructed. To this end awareness and preference of the cat's face were grasped by a survey. Besides, to decide on the proportion of each of the cat's body parts, the proportions of real cats, doll cats and animated cats were researched. To assist a treatment, personified facial expressions and motions were applied to design. According to these guidelines, the design of the cat robot's outer shape was developed.

In order to design the robot's architecture for children with ASD, a rigid support structure should be designed that can express a variety of emotions in the face and body representing various emotions and gestures. To meet these requirements it is required to design the appropriate mechanical structure and controller for making the various motions. In addition, lots of interaction with ASD children is required to design the various types of sensor systems and the operating program for controlling the robot. In this paper, we suggest the cat robot's structures, electrical circuits, and driving program for the treatment of children with ASD.

Material of an existing emotional robot is mostly fake fur or plastic which has an cold and hard touch. Because of the plastic material characteristics, an animal emotional robot which was made of the plastic material does not give the users calmness and pleasure when they commune with the robot. Therefore the robot does not help treatment of children with ASD. Even though the robot material also is fake fur which has feel of actual animal, the users cannot be dodged damage from the harmful environment like house dust mite. So in order to help the emotional therapy as well as protection from 
harmful environment of children with ASD, insecticidal effect against house dust mite by using ethanol extract of three herb teas and cacao was tested.

\section{Need Assessment of Therapists and Parents for Early Cat Robot}

Cross-academic professional clinicians were focus-group interviewed twice and guardians were focus-group interviewed once to investigate the necessary clinical components of the cat robot for the use of early treatment of children with ASD. Two series of FGI records were all recorded and transcribed, and summarized and organized into common categories. Initiatives that use robots to treat ASD children have consistently reported positive results in improved interactions, eye contact, and concentration (Cho, Kwon and Shin, 2009; Feil-Seifer and Mataric, 2008; Lee et al., 2010[5]; Robin et al., 2005, Scassellati, et al., 2012[6]). This study conducted FGIs with professional therapists and parents of children with ASD in order to learn the necessary or desirable motions and functionalities robots should have to be used for treating children with ASD.

\subsection{Expression of Emotion}

- The cat robot must be able to express various emotions

- Various emotions can be expressed with the shape of the eyes, eyebrows, and mouth

- Since eye contact is an especially important measure of interaction, the robot must be able to follow the child's gaze according to the range of eye contact or be able to control the size of its pupil.

It must be able to act and express emotions appropriate to the situations in order to socially behave and interact with children with ASD. As for emotional expressions, they can be generally divided into two strands: positive and negative emotional expressions. These expressions should include joy, sadness, anger, surprise, and various other changes of emotions. Additional auditory effects, if possible, would be desirable. These functions will allow children to experience positive reactions to their own positive behavior and negative reactions to their own negative behavior, bringing about educational and imitational effects for the children, which will help them to become aware of other people's positive and negative responses.

\subsection{Stimulating the Senses}

- The robot must be able to easily express through the various senses depending on the situation (visual, textual, auditory, olfactory)

- Since stimulating the textual or auditory senses is especially more effective for treating children with ASD, the robot must be able to emit color or light and make sounds according to the situation, warm up when there is a lot of contact, stiffen its skin or tail, or express through other textual responses

It must increase social behaviors. The interactions between the cat robot and children with ASD should expedite joint attention. For example, it must be able to make movements that can draw out behaviors such as pointing at the robot or looking in the direction of the robot. It would also be desirable if it could react differently depending on how it is touched. For example, if it is touched softly, it could wag its tails or cry sweetly and if it is touched roughly, then it could raise its tail, scream sharply, or redden its face. It should increase physical contact such as hugging or touching behaviors.

\subsection{Motion Ability}

- Ability to emulate the motions of the child

- Ability to express closeness through motions such as approaching the child or hugging him or her 
- Motioning to request to play with the child

- Ability to move around such as following the child by using wheels or other contraptions

It should be able to move in a way to increase eye-contact or provide other visual stimulus. It should be able to motivate movements such as meeting the eye, holding eyecontact, responding "yes" when called, or turning the head in the robot's direction.

\subsection{Managing the Robot}

- Function for family members or therapists to operate the robot with a remote control

- Ability to select from a various range of programs according to the child's conditions - swappable memory cards

- Function to monitor the changes in the child's behavior throughout the use of the robot

- Therapeutic program and family-use programs both included

- Produced with replaceable robot cover

It needs a function where the robot can imitate the child's actions in order to attract the child's attention. Furthermore, it would be desirable if there was a turn-taking function where the child can wait for the robot's reaction and they can take turns reacting to each other's actions.

\subsection{Material Component}

- The material should be able to be detached and washed

- It should not shed or it should have short hair or made of texturally soft material instead

- Eco-friendly material since it is for children with ASD

- The cat robot's initial model should be developed in terms of design to realize the above components.

Guardians especially hope for the same effects as animal-assisted therapy, such as the role as a robot friend. Additional components that are not therapeutic but may be desirable are realistic considerations such as easiness to wash, safe and durable material, and affordable cost.

\section{Design Development for Early Model of Cat Robot}

Basically, an appearance of cat robot must be looked like one of cat. An appearance of cat is composed of the head, body, arms, legs and a tail. When designing the appearance of cat robot, it need be studied about what the desirable proportion of each part is. Furthermore based on the shape of cat's face among inverted triangle, long and rounded ellipse or round, the feeling could be changed much. So, it must be studied for the preference and recognition as to this appearance's proportion and shape. For making the cat robot more efficient on the therapeutic value, personification must be applied. "Personification" is a design method which is applied not only on robots but also on various industrial products. The main purposes of using personification in industrial design are making a man accept a robot as more familiar, and making it easier to convey the character of a product through the outward appearance or movements to a man. According to a study of Kwak \& $\operatorname{Kim}(2009)$ it is researched that if the features of a robot are more like a friend, warm, and pleasant, the man is more sympathetic to the robot.[7] As a matter of fact, a facial expression of an animal is different from that of a man. But for a man to have sympathy with an animal robot and to give a favorable impression, it needs personification that makes imitation of facial expressions or actions of a man to some extent. Therefore, for the cat robot to take several personified actions, there need many sensors and driven 
motors. Due to these environmental conditions, the proportions of cat robot could change, compared to the real.

\subsection{Guidelines for Design of Cat Robot's External Features}

For the design development of the cat robot's external features, guideline had to be established, i.e., shape, structure of face, arms and legs, body and tail, etc., and interaction of acts. Thus, preference and awareness surveys were performed. Using computer graphics in which the cat's face size, ears, and eyes were transformed. The result of the survey using the image board showed the most popular cat image had big ears, and centered eyes. Therefore, to design a cat robot, preferred by children, it must have big ears and centered eyes.
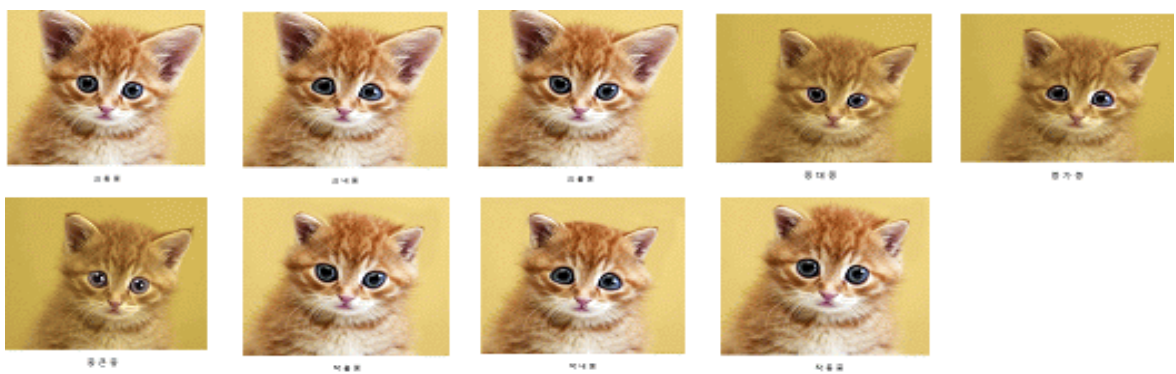

Figure 1. Transformed Face of a Cat using Computer Graphics

To decide on the proportion of each of the cat's body parts, the proportions of real cats, doll cats and animated cats were researched. In real cat, in the case of kittens, the proportion of the head to the body is about 1:3, compared with that of cats, which is about 1:5. In the case of dolls or characters, for an emphasis on cuteness, kittens and cats both have a proportion of $1: 1$. Therefore, the cat robot must be made with a proportion of 1:2, to emphasize the kitten's cuteness but make it feel like real cat.
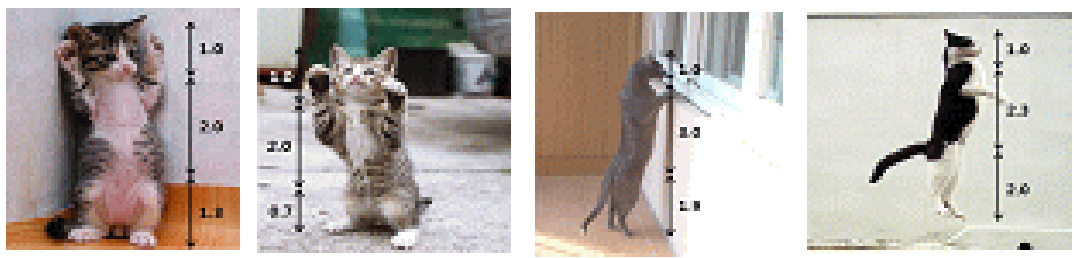

Figure 2. Proportions of Real Cats
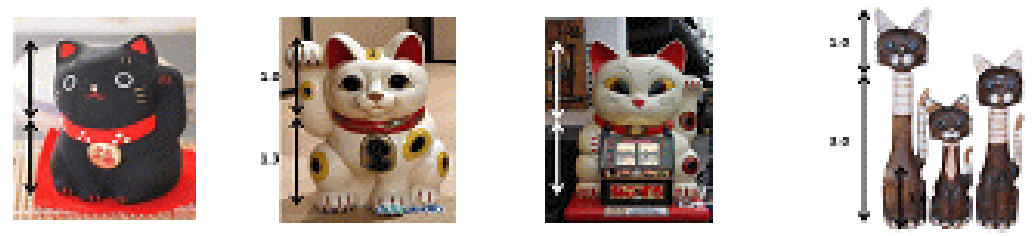

Figure 3. Proportions of Doll Cats

The size of the ears, the shape of the eyes, the size of the mouth and the proportions between them were researched in the face of a cat. For children to recognize the face of the treatment robot as a cat, it should be designed considering the proportions of real cats. To make it like a character it should have larger ears, centered eyes, and an actual sized mouth that is emphasized to express the changes of facial expression. Personification of animal robot is personified to increase a familiarity and awareness of function and character a human. Emotional animal robots are domestic 'Kobie'[8] of ETRI (Korean 
Electronics and Telecommunications Research Institute), entertainment pet dog robot 'Aibo'[9], a cure robot shaped seal pups 'Paro'[10] of AIST, made just like a space animal 'Furby'[11] etc. There are two ways of represent facial expression. The first is a way to make facial expression by moving muscles, and the second is to show emoticon or eye shape by the use of LCD.

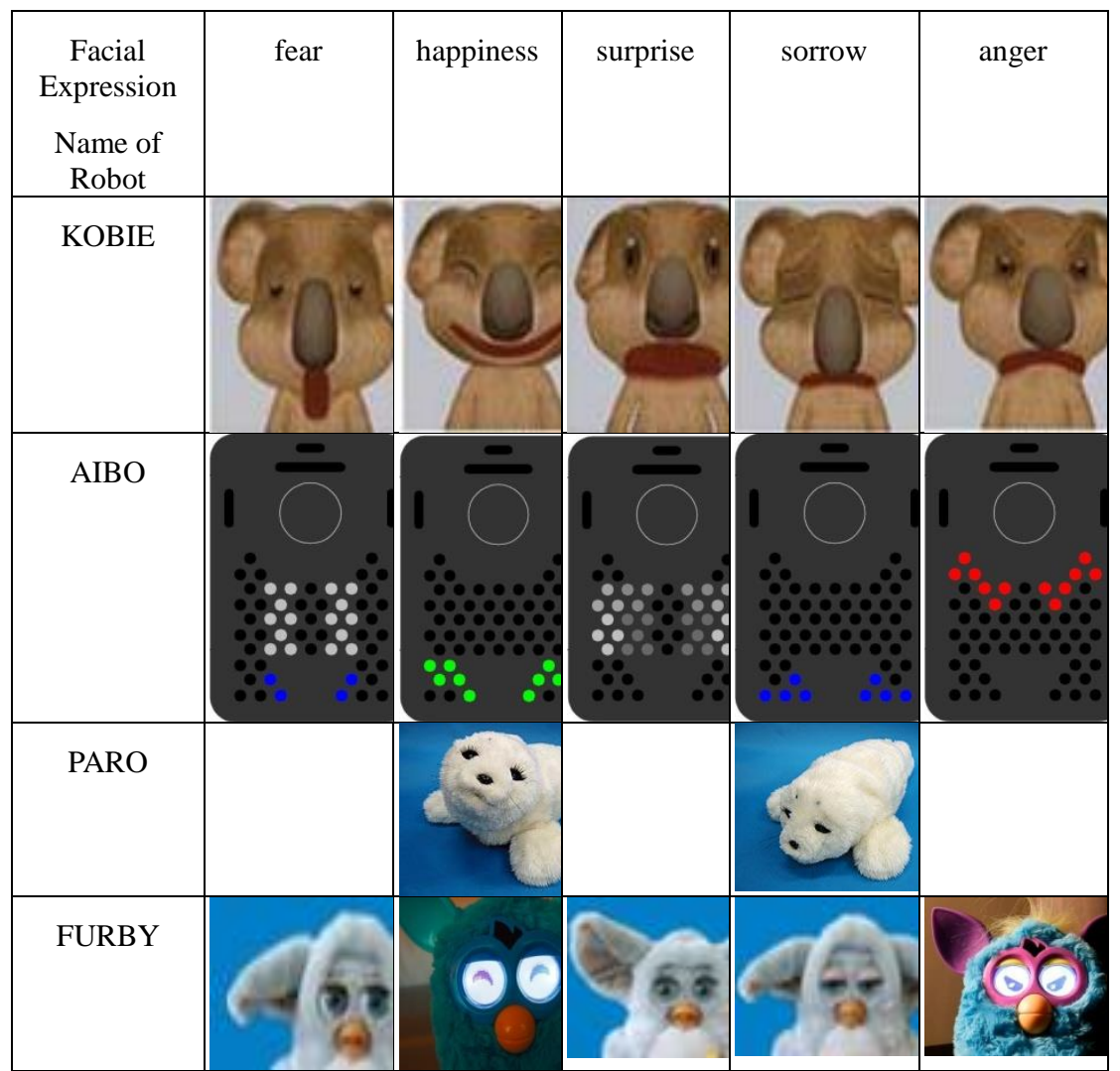

Figure 4. Facial Expression of Animal Type Robot for Personification

\subsection{Behavior Design of a Cat Robot}

For the design of a cat robot, cat's action must be concerned first of all. A cat should have various emotions and show her emotions with whole body such as facial expressions, tail, ears, acts and etc. When a cat comes close with her tail upright, it means 'I'm hungry, please feed me.' When a cat feels scared, she suddenly rolls her tail into inside of her body, or on the contrary she makes her tail big to be lift up. In addition, when a cat shows curiosity looking at something, she moves her tail-end slowly. But, for the early treatment of children with ASD, not only behavioral act of a cat but also personified act such as 'hugging', 'eye contact', 'imitating body movement' and 'reaction to negative acts' etc. must be applied. To hug like a man, the first pose of a cat robot must be shaped in a seating posture with her hind legs stretched forward and her forelegs upright. 

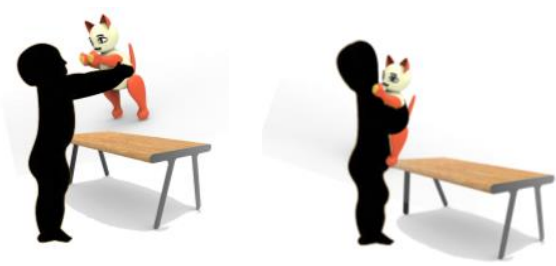

'Hugging'
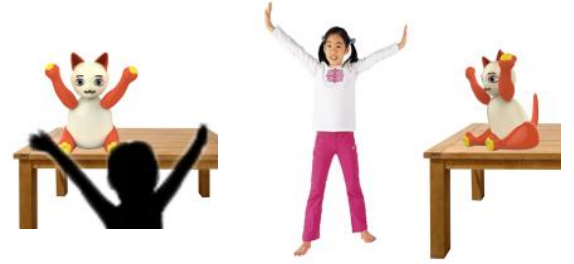

'Imitating Body Movement'
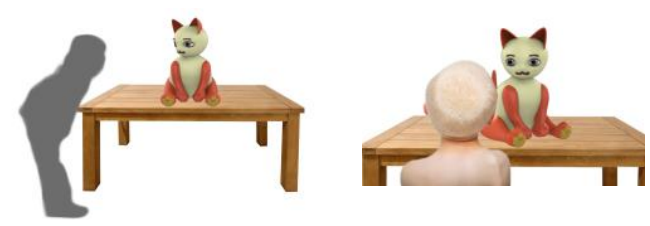

'Eye Contact'

\section{Figure 5. Embodied Actions by 3D Computer Graphics}

The mouth of a real cat is rather small but the mouth of a cat character is very large, because it is used as a means of personification. In the case of the treatment cat robot to improve awareness it needs to emphasize changes of facial expression and it needs to be personified. Therefore, the size of the mouth should be exaggerated, and moving the mouth up and down should direct various facial expressions. For the treatment of ASD children happiness, sadness, anger and surprise should be expressed with the personified facial expression. According to the experts say the actions of the cat robot included eye contact, hugs, the copying of body movements, and reaction to inappropriate acts.

\subsection{Character Design of a Cat Robot}

The character of a cat robot, for the early treatment of children with ASD, needs to be personified for looks like not a cat's character but a human's character (Disalvo, Gemperle, and Folizzi, 2004). Applying personification which projects a shape of an animal or a human onto the shape of a robot has a man come close to a robot more friendly, and makes the character of a robot to be delivered to a man through an appearance or movement in an easier (Disalvo, Gemperle, and Folizzi, 2004). [12] When applying the personification of a cat robot, the character of both an animal and a human must be considered. Because, in the animal personification, non-verbal communication area such as shape of appearance, non-verbal act of a man and behaviour act of an animal are very important. The important elements that reveal the character of a cat are a direction of expressions and a handling of eyes. The expression of both an animal and a human presents an internal emotion. The facial expression of a real cat is different from a man. Only a man who feeds a cat can understand the facial expression of a cat, because they will sympathize with the cat. They understand the cat because they always observe the cat, others cannot understand with ease. The eyes of cat become thin when a cat is satisfied, and look sharp because the tale of cat's eye is a slanted line. On the other hand, the shape of man's eyes is thin but the upper part is round. Therefore, it can be confirmed that on the case study of animal robots like Kobie and Furby, their eyes are personified to the shape of man's eyes. For the expression of satisfaction, if the eyes are expressed like a real cat, a man who does not know about the emotional expressions of a cat could misunderstand that a cat is angry. Because as in the above case study, when angry Furby and Eibo's eyes are the same as satisfied cat's eyes. So facial expression of a cat robot 
must look like the personified man's facial expression, rather than look like the facial expression of a real cat.

\begin{tabular}{|l|c|c|c|}
\hline Expressed emotoin & Satisfaction & Exciting & Angry \\
\hline $\begin{array}{l}\text { Facial expression of } \\
\text { real cat }\end{array}$ & Happines & Surprise & Angry \\
\hline $\begin{array}{l}\text { Expressed emotoin } \\
\text { expression of a cat } \\
\text { by 3D rendering }\end{array}$ & & & \\
\hline
\end{tabular}

Figure 6. Compare with the Facial Expression of a Real Cat and Personified 3D Rendered Cat

Lee(2003) analyzed the existing studies to abstract a fear, an anger, a sorrow, a happiness and a disgust as common emotions of a man, to develop the tool for the management of robot's reacting motion.[13] On this study, it would be proceeding with five emotional expression of a cat robot substituting a disgust with an interest. With regards to the early treatment of children with ASD, because of the expression with simple and basic essentials would be considered more effective than too much emotional expression.

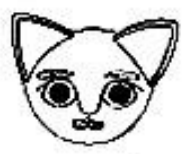

normal

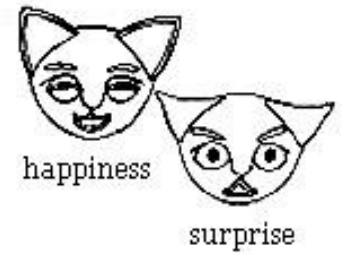

surprise

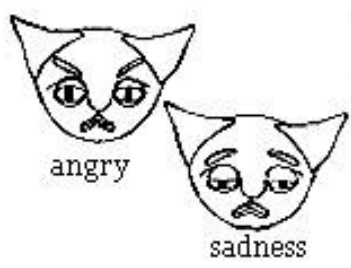

sadness

Figure 7. Facial Expressions of Cat Robot for Design

\subsection{Process of Design Development of a Cat Robot}

To make an early working model of a cat robot for the treatment, various sketches were developed. The usual figure of a cat robot was set up as a figure of seating posture with her hind legs stretched forward and her forelegs upright. As you see, it was the easier posture to make an eye contact/hug with the children and follow the motion of children. The usual expression of a cat robot was set up as soft, friendly and tender. Because, it was studied that the warmer and tender of the robot's appearance like a friend, the more gain the sympathy. (Kwak and Kim, 2009) As an emotional concern for the treatment, the cat was sketched with normal length fur to make it feel like a real cat. The proportion of the face and body was given by 1:2 to express the cuteness of a kitten. To show changes of 
facial expression, eye brows were emphasized, eyes were made bigger than normal size, and the eyes were centered according to the results of the preference research. Figure 4. A, $\mathrm{B}$, and $\mathrm{C}$ show the three sketches developed to match these criteria.
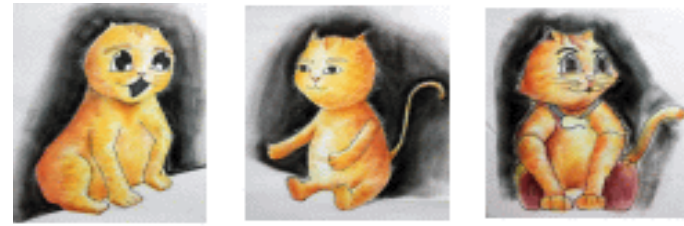

Figure 6. Sketches A, B, and C

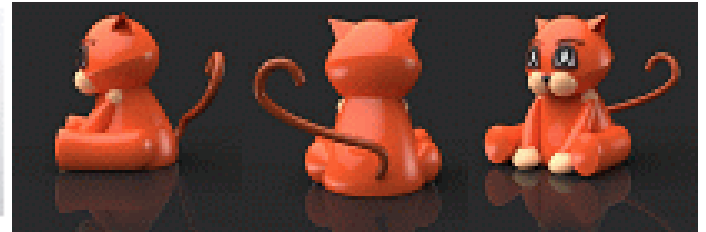

Figure 7. 3D Rendering of Sketch C

Of the three proposed sketches the most suitable sketch $\mathrm{C}$ was processed by 3D rendering. This rendering was used by the department of engineering as the outer shape of the cat robot for shape of the cat robot for the interior design. The cat robot's sitting height was set at $50 \mathrm{~cm}$ to match the eye level of children. In still included parts that were partially disconnected, but the positions of the inner motors for moving the eyes, eyebrows, ears, arms, and legs were drawn.
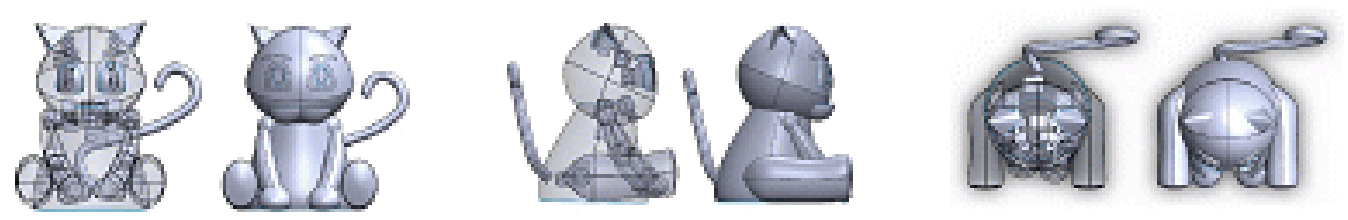

\section{Figure 8. Front View, Side View, and Top View of the Drawing Include Inner Motor's Positions}

To make a final proto-type model, the early 3D rendering was corrected. To make it look more like a cat, the shapes of the face, ears and body were reformed and to improve the facial expression of the cat robot, the shapes of the eyes, mouth and ears were corrected. The 3D rendering for the second interior design was developed. According to this $3 \mathrm{D}$ rendering the second interior design was developed by the engineering department as seen in Figure 9.

A referred cat robot was designed to be covered by furry coat with the same length of furry as popular one to be felt like a real cat in consideration of emotion. The Proportion between a face and a body should be given with 1:2 to show the cuteness of a baby cat. For a better showing of the change on a facial expre- ssion, eyebrows were emphasized and eyes were made bigger than normal size according to the result of a preference research and tails of eyes were centered. The size of a cat robot are designed with the height of $50 \mathrm{~cm}$ to be set up at the children's eye level as shown in the Figure 10.

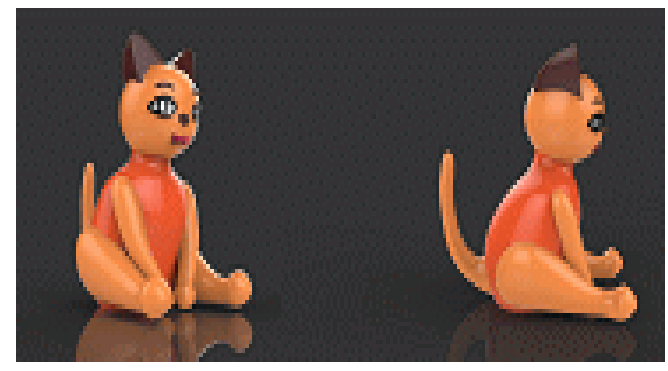

Figure 9. Corrected 3D Rendering 

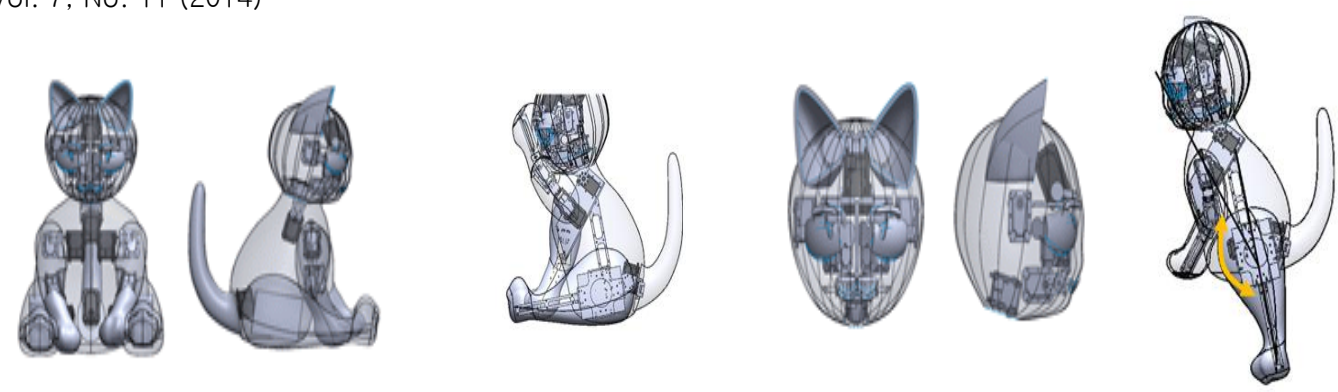

Figure 10. 2nd Internal Mechanism Drawing

To put it together using each part's holes that were prepared during the interior engineering design, the exterior design of the head was developed by dividing it into two parts, front and rear using Rhino as a tool.

Body of the cat robot divided into two parts and the back part has another cover for the change of battery, cable, censor etc.
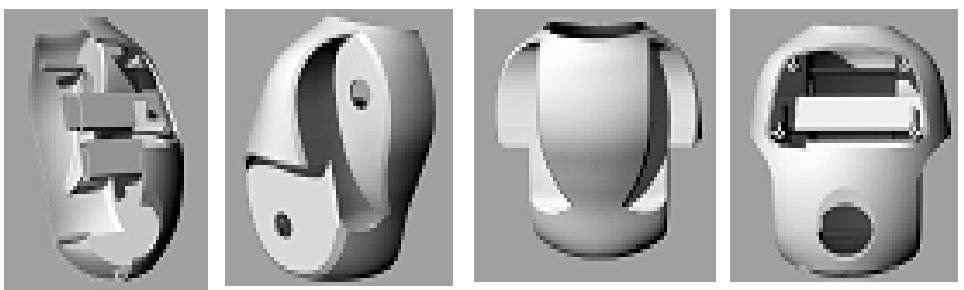

Figure 9. Body of the Cat Robot made by 3D Printer
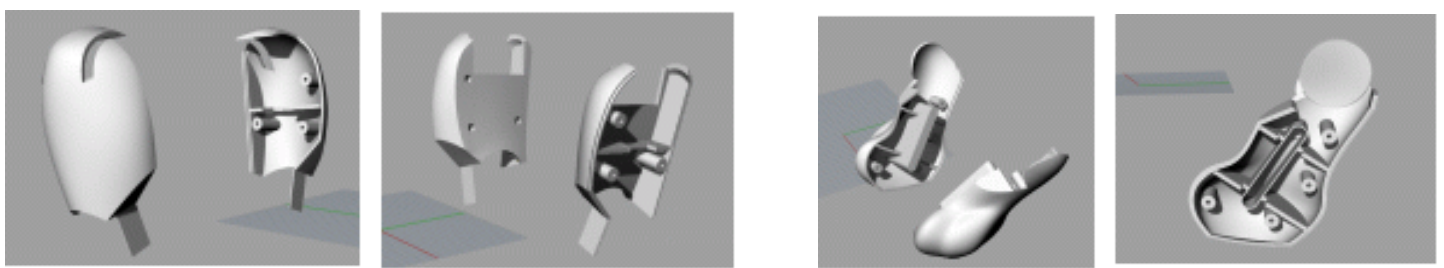

Figure 10. Shoulders and Arms Inner and Outer Part

Arms divided into two parts as upper part in which include shoulder and lower part. Arms are drawn to cover as possible when arms are folded and unfolded not to seen inner parts. And they are divided into two parts of right and left to possible put together.
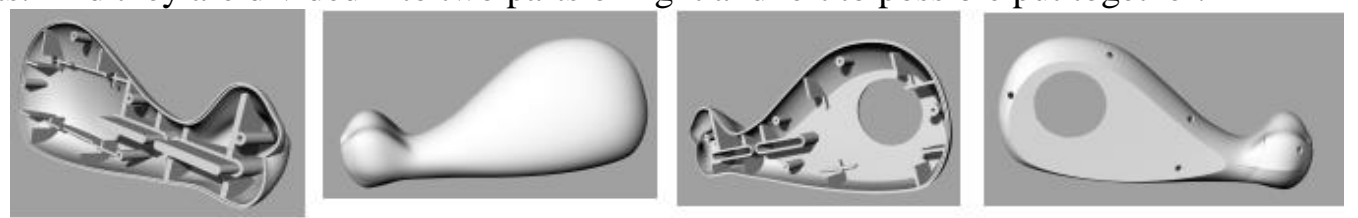

Figure 11. Legs (Right and Left - Inner and Outer)

Legs are divided into two parts and make a hole to connect the inner part of the body. 

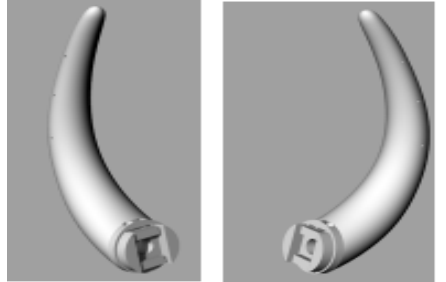

Figure 12. Tail

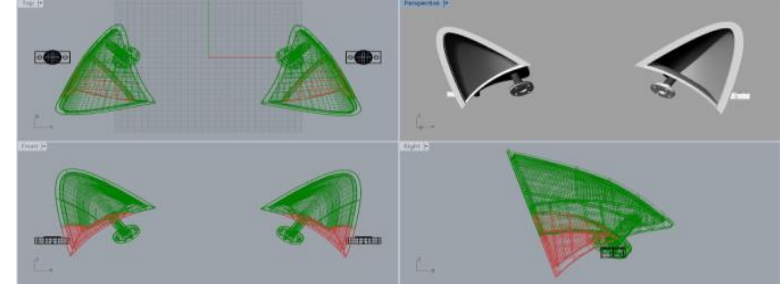

Figure 13. Ears

Tail is made of the structure with honeycomb to raise the strength. Ears should be lied on its side and folded according to the facial expression of cat robot. If the part of folded is too hard it should be bored of the part of head too much. As a result many parts of the inner side are to be seen. Therefore the part of marked red is made by soft material such as silicon or rubber it could be folded.
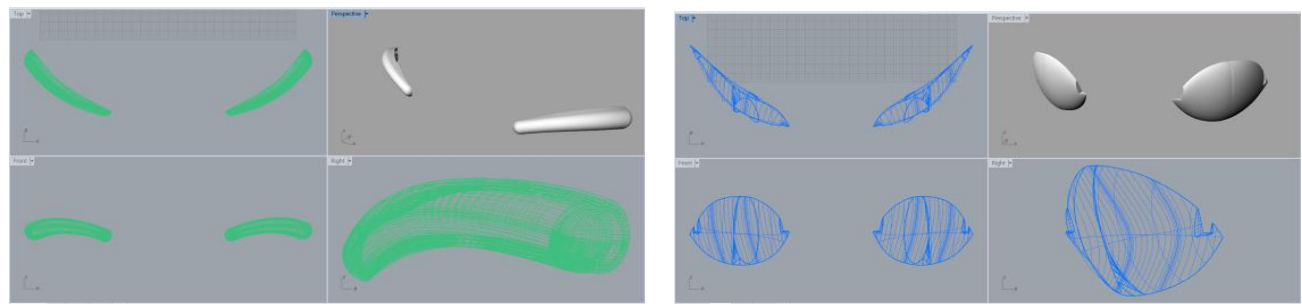

Figure 14. Eyebrows and Eyeballs

Eyebrows are designed to possible the area of movements suggested by inner plan. Eyeballs should be existed in eyelids and should be made of transparent material to present pupil of the eyes by graphic work.
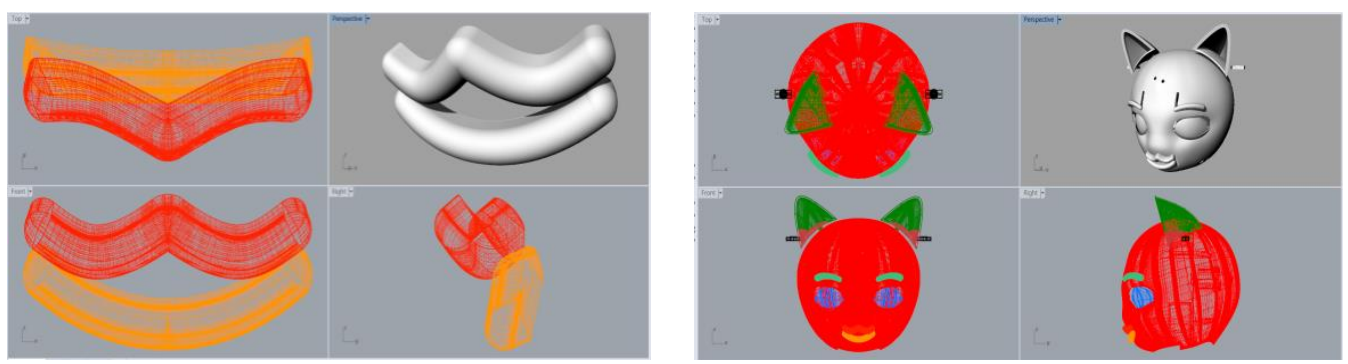

Figure 15. Lips and Face

Oral angle of lips should be moved up and down in the range of the movement suggested by inner part of engineer to present the change of facial expression. So it should be used by soft material such as silicon or rubber.

\section{Electrical System Design}

\subsection{Joint system Design}

Cat robot for the treatment of children with ASD should be considered the action against the user's abnormal behavior. For the treatment of emotional expression to a face part, intelligent controller units related with the movement should be considered in the body portion and the tail portion. It maybe takes after real cat and be able to do similar movement. Head unit is very important to represent the emotion. It has parts of the eyes 
with forehead, eyebrows, mouth, and ears like a cat, which are operated with 10 motors. Furthermore the each parts of the face are arranged separately and controlled by each individual motors, so they can move independent and express the required emotions. In connecting the head and neck of the body, two degrees of freedom is implemented with the motion of shaking and nodding, so cat robot has a total of 21 degrees of freedom.
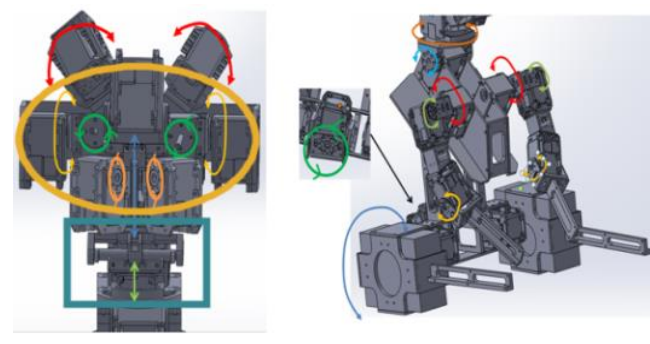

Figure 11. Motor Movements in Body and Face

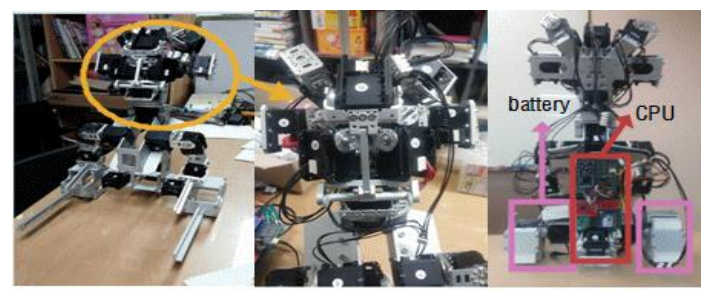

Figure 12. Cat Robot Skeleton and Motor Installation

Generally cat robot has an initial posture sitting at table, and be excluded the walking function of real cat. It just vents the main body using hip joints to make body motion. Especially hip motors are so powerful enough to drive the body and withstand the load variation from external. In addition various types of sensor system were installed on the skin or under the skin in order to give robot-human interaction such as intelligent behavior and sympathetic action.

\subsection{Controller Design}

The operations of each part of the body and interactions between robot and ASD children are detected by wide range of internal and external sensors of the robot. Several capacitive contact sensors were installed on head, back, belly, and each arm and leg, which can detect the user's touch. In addition sound generating device was designed to generate cat voice according to various situations. The cat sound can be reproduced through audio Codec with electrical speaker. Sound effect is essential to make interaction each other, especially ASD

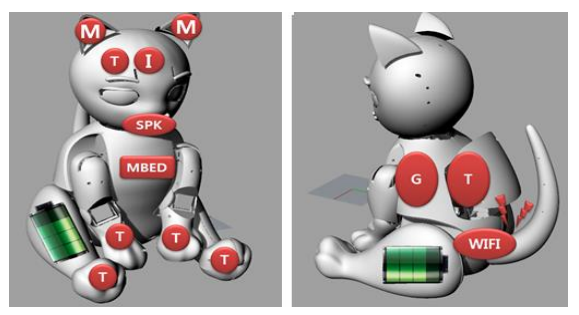

Figure 13. Sensor and Actuator Deployment 


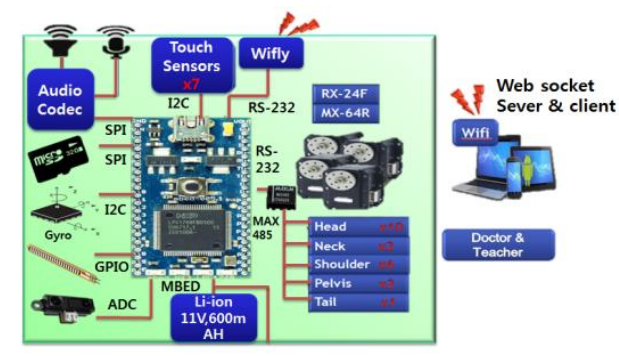

\section{Figure 14. Block Diagram of the Controller}

treatment. An infrared sensor can catch the motion when somebody moves within a boundary of certain distance. It can be able to measure the distance between user and robot and also direction of movement, so it can be used to increase intimacy with robot. In the waist, a 3-axis accelerometer was installed to measure the robot orientation if the robot was tilted, lifted, or if user try to hug according to the user's feeling.

All the proposed motion is determined by number of commands that ordered from operator on the web through Wireless Internet (Wifi), and robot behavior and actions is set by downloading command on the web. In this way a large number of robots operating in wide field are available to play in the response of the robot and easily collect data as a useful therapeutic effect, and the teacher or operator can control the robot remotely as a medium of treatment.

\section{Conclusion}

A cat robot for an early treatment of children with ASD should have to look like a real cat for understanding as a cat to children with ASD. On the other side it needs to be exaggerated expression by personification for better understanding of motions or facial expressions. Early model of cat robot's outward shape, motions and facial expressions were designed, planned and produced according to these requirements. The therapeutic components required of a cat robot for the use of early treatment of children with ASD are the ability to express various emotions, to carry out social behaviors, to hold eye contact with the children, and to imitate actions.

Joint movement, facial expression, and sensory system were proposed in order to realize their function including how to install and control the body. The proposed robot will have 8 joints in the body, 8 joints in the face, and be deployed over than 10 kinds of sensory system with sound management and touch detection.

An effort to sophisticate this early developmental model must be continued. Follow-up studies that take this early developmental cat model and evaluate its effectiveness and improve its weaknesses through actual experiments should be carried out. This early model of cat robot should have to be covered with appropriate artificial furry coat. In addition the cat robot should be corrected by feedback through realistic application and experiment to children with ASD and normal children. These kinds of studies using corrected perfection model of animal robot is expected to help early treatment of children with ASD.

\section{Acknowledgement}

This work was supported by the National Research Foundation of Korea Grant funded by the Korean Government (NRF-2013R1A2A2A04014808).

This paper is a revised and expanded version of a paper entitled [Development of Design of Initial Cat Robot Model for the Use of Early Treatment of Children with Autism Spectrum Disorder (ASD)] presented at 2014 ICHCI Conference, Budapest Hungary, Aug 14-17. 


\section{References}

[1] K. Cho, J. Kwon and D Shin, "Trends of cognitive robot based intervention for autism spectrum disorder", Journal of Korean Association for person with autism, vol. 9, no. 2, Journal of Korean Association for person with Autism, (2009), pp 45-60.

[2] B. Robins, K. Dautenhahn and R. Te Boekhorst, "Billard A: Robotic assistants in therapy and education of children with autism: Can a small humanoid robot help encourage social interaction skills?", Univers. Access Inf. Soc., vol. 4, no. 2, (2005), pp. 105-120.

[3] D. Feil-Seifer and MJ Matari'c, "B3IA: a control architecture for autonomous robot-assisted behavior intervention for children with autism spectrum disorders", Proc. 17th IEEE Int. Symp. Robot Hum. Interact. Commun, (2008), Aug. 1-3; Munich, Ger.

[4] K.H. Kim, H.S Lee, S.J. Jang and H.J. Ku, "The Reaction of Children Labeled with Autism through Interactions with Robot", Journal of emotional and behavioral disorder, vol. 26, no. 1, (2010), pp.331353.

[5] H. S. Lee, S. S. Baek, H. J. Ku, W.S. Kang, Y. D. Kim, J. W. Hong and J.U. An, "Experimental Research of Interactions Between Children with Autism and Robots", Journal of Emotional \& Behavioral Disorders, vol. 26, no. 2, (2010), pp. 141-168.

[6] B. Scassellati, H. Admoni and M. Mataric, "Robot for use in autism research, Annu", Rev Biomed Eng, vol. 14: (2012), pp. 275-294.

[7] S. Kwak and E. Kim, "Design of the Emotional Robot for Human Empathy", Journal of Korean Society of Design Science, vol. 22, no. 5, (2009), pp. 27-34.

[8] Tagstory, http://www.tagstory.com/video/100151689

[9] You Tube KR, http://youtu.be/212P8Uz0LkA

[10] Seal Type Mental Commit Robot PARO, http://www.paro.jp/english/function.html

[11] You Tube KR, http://youtu.be/IAZ8QG8uz9I

[12] C. Disalvo, F. Gemperle and F. Folizzi, "Imitating the human Form: Four kinds of Anthropomorphic Form", Futureground 04 Proc, (2004).

[13] D. K. Lee, "A Study on the Entertainment Robot Behavioral Structure and Reactive Motion Design", KAIST, Master's thesis, (2003).

\section{Authors}

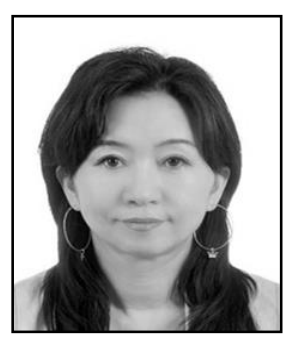

\section{Keum Hi Mun}

1998-2014 Professor, Dept. of Industrial Design, Semyung University, Jecheon, Korea

1993-1998 Prof. of Seoul Polytechnics College, Dept. of Industrial Design / Seoul, Korea

1987-1992 Designer of Ssangyong Motros Co., Pyungtaek, Korea

1982-1983 Designer of Kia Motors Co., Seoul, Korea

2007-2010 Complete Doctoral Course of Western Philosophy in Hungbuk University / Chungju. Korea

1994-1996 Master of Industrial Design in Hongik University/Seoul, Korea

1975-1979 Bachelor of Industrial Design in Seoul National University/Seoul, Korea

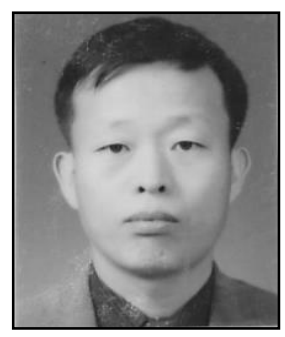

\section{Bo Hee Lee}

1997-2014 Professor, Dept. of Electric Engineering, Semyung University, Jecheon, Korea

1981-1985 B.S. degree in Electronics Engineering from Inha Universit

y/ Incheon, Korea

1991-1996 M.S. degree and the Ph.D. degree in Electronic Engineering from Inha University, Incheon, Korea 
1984-1987 Researcher at telecommunication system of Samsung Electronics Co. Ltd., Seoul, Korea

Main research interests: areas of automation, modular robotics, and embedded system applications

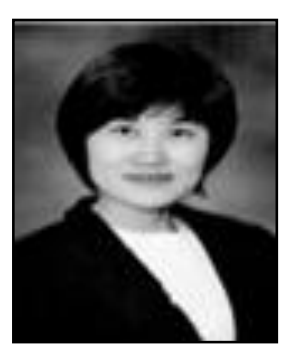

\section{Ja Young Kwon}

2010-2014 Professor, Dept. of Social Welfare, Semyung University, Jecheon, Korea

2010-2005 Ph.D. in Social Welfare, Ewha Womans University Seoul. Korea

2003-2009 Director of Secho Mental Heath Center/ R. O. Korea

1999-2003 Psychiatric Social Worker at Samsung Seoul Medical Center/ R. O. Korea

1990-1993 Psychiatric Social Worker at Hangang Sacred Heart Hospital/ R. O. Korea

1991-1989 M.A. in Social Work in Ewha Womans University, Seoul. Korea

1985-1989 B.A. in Social Work in Ewha Womans University, Seoul. Korea

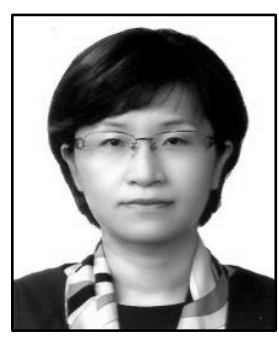

\section{Jin Soun Jung}

2001-2014 Professor, Dept. of Fashion Design, Semyung University, Jecheon, Korea

1983-1987 Bachelor of Home Economics from Pusan National University, Pusan, Korea

1987-1989 Master of Home Economics from Pusan National

University, Pusan, Korea

1992-1997 PhD in Science from Pusan National University, Graduate School, Pusan, Korea 
International Journal of Control and Automation Vol. 7, No. 11 (2014) 